\title{
Hypoactive Sexual Desire Disorder
}

National Cancer Institute

\section{Source}

National Cancer Institute. Hypoactive Sexual Desire Disorder. NCI Thesaurus. Code

C94337.

A disorder characterized by a recurrent or persistent lack of desire for sexual activity. The lack of sexual desire is not attributable to another psychiatric disorder or to the physiological effects of substance use or a general medical condition. 